IOS Press

\title{
Introduction to the thematic issue on Mobility
}

\author{
Christoph Stahl ${ }^{\mathrm{a},{ }^{*}}$, Bernd Krieg-Brückner ${ }^{\mathrm{a}}$, Wolfgang Zagler ${ }^{\mathrm{b}}$ and Björn Göttfried ${ }^{\mathrm{c}}$ \\ ${ }^{a}$ Cyber-Physical Systems, German Research Center for Artificial Intelligence, DFKI GmbH, \\ Enrique-Schmidt-Straße 5, D-28359 Bremen, Germany \\ ${ }^{\mathrm{b}}$ Institut für Gestaltungs- und Wirkungsforschung, Zentrum für Angewandte Assistierende Technologien, \\ Favoritenstraße 11/187-2b, A-1040 Wien, Austria \\ ${ }^{\mathrm{c}}$ Artificial Intelligence Research Group, Centre for Computing and Communication Technologies, University of \\ Bremen, Germany
}

\section{Introduction}

This thematic issue of JAISE focuses on mobility assistance solutions that help users navigate from one place to another in everyday life, as pedestrians indoors and outdoors or by car. Particularly diminished physical (visibility, hearing, strength, balance) and cognitive (moderate dementia) abilities can seriously affect individual mobility as persons feel unsafe and disoriented. They prefer to stay at home in their familiar environment, neglecting their own fitness, health condition, and social contacts, and in the worst case suffer from depression. In order to support an independent and healthy lifestyle, numerous research projects are currently investigating various aspects of mobility assistance. Most of them apply concepts related to Ambient Intelligence in mobile scenarios, particularly tailored towards the special needs of elderly and impaired users (Ambient Assisted Living). In addition, young and healthy users can benefit from intelligent route planning algorithms and indoor wayfinding assistance.

Current navigation solutions, as provided by smartphone apps and cars, are dedicated to help answering the questions "Where am I?" using GPS and "What is the route to my destination?" using street maps. The Guest Editors of this JAISE thematic issue, Christoph Stahl, Bernd Krieg-Brückner, Wolfgang Zagler and Björn Gottfried, aim to investigate mobility assistance in a much broader scope and sought papers that present interesting solutions for various aspects of mobility, e.g. advanced route planning algorithms, mapping of indoor and outdoor environments, interaction

*Corresponding author. E-mail: christoph.stahl@dfki.de. design for wayfinding, indoor and outdoor positioning techniques beyond GPS, and motion planning methods for physical support platforms, such as robotic wheelchairs and walkers.

The development of innovative mobility assistance systems requires individual solutions for all of the aforementioned aspects, which makes it particularly challenging. Consequently, the papers presented in this thematic issue apply methodologies and technologies from a broad range of research areas, i.e. HumanComputer Interaction, Ubiquitous Computing, Artificial Intelligence, Geoinformatics, Computer Vision, and Robotics.

The selected papers will give the reader an overview of different strands of research and familiarize the reader with the state-of-the-art in mobility assistance. The next section briefly summarizes each paper in this thematic issue, putting it into context.

\section{In this thematic issue}

Route planning is at the core of any navigational aid, and current products usually offer only the shortest and fastest route towards a destination. This thematic issue contains two approaches that consider points of interest and constraints along the route. Shin, Ziebart and Dey propose in their paper entitled 'Serendipityempowered path planning for predictive task completion' a path recommendation system that helps commuters to overcome the difficulty of managing everyday tasks during regular driving. The system generates a number of paths by extending routes to consider the given tasks of drivers and corresponding point of interests and ranks them by their quality. Using the best path, users are able to perform their daily tasks by 
serendipity (fortunate occurrence) on the way to their originally planned destination.

Colombo et al. focus in their paper 'Efficient customisable dynamic motion planning for assistive robots in complex human environments' on people with physical and mental impairments that require the support of a walker in indoor environments. Such users often find it challenging to navigate in crowded or unfamiliar environments. The authors present a motion planning algorithm that is able to intelligently deal with crowded areas, anomalies (road blocks, wet floors, ...) as well as constraints ("keep a toilet within reach of 10 meters during the journey", "always avoid stairs", ...).

Route finding algorithms require digital models of the environment, such as street maps. Today, global companies maintain and provide geospatial databases for satellite navigation systems worldwide. Regarding indoor spaces, there is no such general provider for maps; each building must be individually modeled. Ohm, Müller and Ludwig introduce in the paper entitled 'Displaying Landmarks and the User's Surroundings in Indoor Pedestrian Navigation Systems' a map modeling toolkit for built environments that supports indoor routing and the generation of graphical route descriptions based on 'functional' landmarks, such as stairs or doors. Based on the toolkit, the authors developed an indoor navigation app and evaluated the usability of detailed maps compared to abstract graph-like depictions of the route.

The following two papers address the special needs of visually impaired or blind people. The usual tool for the blind, the white cane, is helpful but does not provide much information about obstacles or the path to follow that must be memorized exactly by the user. Hence the development of navigational aids for the blind has been an active area of research for decades and is now driven by smartphone technologies. In particular, the limited accuracy of GPS is not sufficient to safely guide visually impaired users on the side- walk, so that better positioning methods are sought. Tapu, Mocanu and Zaharia introduce in their paper 'ALICE: A smartphone assistant used to increase the mobility of visual impaired people' Computer Vision-based techniques to detect and classify in realtime static and dynamic objects without any a priori information about the obstacle type, size or location. The device offers an acoustic feedback that warns the user of the presence of any type of obstacle situated in their near surrounding. The system also enhances the positioning method by fusing GPS and inertia sensors with the recognition of landmark images. As a novelty, ALICE is fully integrated into a regular smartphone.

A similar approach is pursued by Jonas et al. in the project 'IMAGO: Image-guided navigation for visually impaired people' that utilizes the camera of a chest-mounted smartphone to record individual routes in urban environments. The stream of images is processed on a server where a 3D point cloud is reconstructed using a structure-from-motion technique. Afterwards, the user can choose a prepared route for navigation. The smartphone takes pictures of the environment, which are sent to the server for image-based localization, orientation and navigation. The direction to walk is sent back and passed to the user via a tactile arrow on the I-Cane device.

\section{Acknowledgements}

The guest editors wish to thank all the reviewers for their valuable contributions to this thematic issue and for helping us to find experts in all areas of Computer Science that were covered by the submitted papers. Additionally, we gratefully acknowledge the outstanding and constant support and guidance of the editorial staff of JAISE. Finally, we would like to express our great appreciation to all the authors for their submissions of high quality that made this thematic issue possible. 